\title{
Magnetic Design of a Superconducting Toroidal Gantry for Hadron Therapy
}

\author{
Enrico Felcini, Luca Bottura, Jeroen van Nugteren, Gijs de Rijk, Glyn Kirby and Bertrand Dutoit
}

\begin{abstract}
Hadron and proton therapy are cutting edge techniques for cancer treatment and a great development of specialized medical centers and research facilities is foreseen in the next decades. One of the main obstacles to the penetration of the use of charged particles for therapy is the construction of complex and expensive accelerating structures and rotating transfer lines, i.e. gantries, able to bend and focus the beam down to the patient. GaToroid is a novel concept of a fixed toroidal gantry, able to deliver the dose at discrete angles in the whole range of treatment energies in steady-state configuration. The steady-state current and magnetic field are appealing features, implying simplified demands on stability, powering, mechanics and cooling, as well as for the clinical perspective, allowing rapid variations of beam energy and treatment angle. In this work, we present the magnetic design of the toroidal coils composing the first instance of GaToroid, focusing the analysis on an option for a proton machine with an energy range of $70 \mathrm{MeV}$ to $250 \mathrm{MeV}$. To create a proper magnetic field distribution, the coils have been designed with peculiar asymmetric shape and the windings have been graded. An initial winding geometry was obtained with an optimization aiming at maximum energy acceptance of the gantry. We are now progressing to the detailed engineering design. We describe here the overall magnet design, coil and conductor layout (LTS and HTS options), and mechanical studies involving the general torus structure. Quench protection is evaluated for LTS (Nb-Ti) configuration, as well as more innovative HTS (ReBCO) options. Finally, we present the design and the construction of a scaled-down demonstrator, intended as the proof of principle of winding procedure and mechanical coil structure.
\end{abstract}

Index Terms - Gantry, Hadron Therapy, Magnet Design, Superconducting Coils, Toroidal Magnets

\section{INTRODUCTION}

$\mathrm{H}^{2}$ adron therapy is a cutting-edge radiation therapy which makes use of heavy particles (protons and ions), to deliver highly localized dose to tumors, minimizing potential damage to healthy biological structures. By varying the particles energy, it is possible to modify the Bragg peak position and thus deliver the required dose at different depths into the patient. Hadron therapy relies on accelerating structures, as well as a beam transfer lines, able to bend, confine and control the particle beam from the accelerator down to the patient [1]. To further ameliorate the effectiveness of hadron therapy, the radiation can be delivered through various angles around the tumor using a rotating transfer line, i.e. a gantry. Hadron therapy centers have high cost, deriving, among others, from

Manuscript received September 24, 2019. This Project has been funded by the CERN Budget for Knowledge Transfer to Medical Applications.

E. Felcini, L. Bottura, J. van Nugteren, G. de Rijk and G. Kirby are with the TE Department, CERN, Geneva, Switzerland (e-mail: enrico.felcini@cern.ch).

B. Dutoit is with École Polytechnique Fédérale de Lausanne, 1015 Lausanne, Switzerland the footprint of the gantry and from the size of buildings required to house the related structures [2]. Classical solutions for hadron therapy, based on rotating transfer lines, are bulky and result in large, heavy and expensive infrastructures. This is especially true for heavy ions gantries, such as Carbon ions, characterized by a diameter larger than 10 meters and more than 600 tons of weight [3]. Superconducting magnets have been used to increase the achievable magnetic field and, therefore, to reduce size and weight [4]. However, the rotating nature of the gantry introduces significant complexity in terms of cryogenics and mechanical stability. Here we present a fixed toroidal gantry, i.e. GaToroid [5][6], able to deliver the dose at a discrete number of angles with neither rotation of the magnets nor the patient. The basic principle is to use the axissymmetric magnetic field between each pair of coils constituting the torus to bend and focus accelerated particles down to the isocenter. If proven successful, the idea of a steady-state toroidal gantry, realized with superconducting magnets, could result in a quantum step toward the concept of single room facilities for hadron therapy.

\section{Coll Optimization}

The GaToroid working principle is explained in detail in [5][6], together with analytical approximations used for the coil geometry and magnet design. Its basic principle is to use a toroidal field (toroidal gantry) to direct beams of different energies and directions on the same point (isocenter) by acting on a single upstream bending magnet (vector magnet). The work described in this manuscript is focused on a configuration for proton treatments. Fig. 1 presents an artistic view of a 16 coils GaToroid for protons, with the patient in the treatment position.

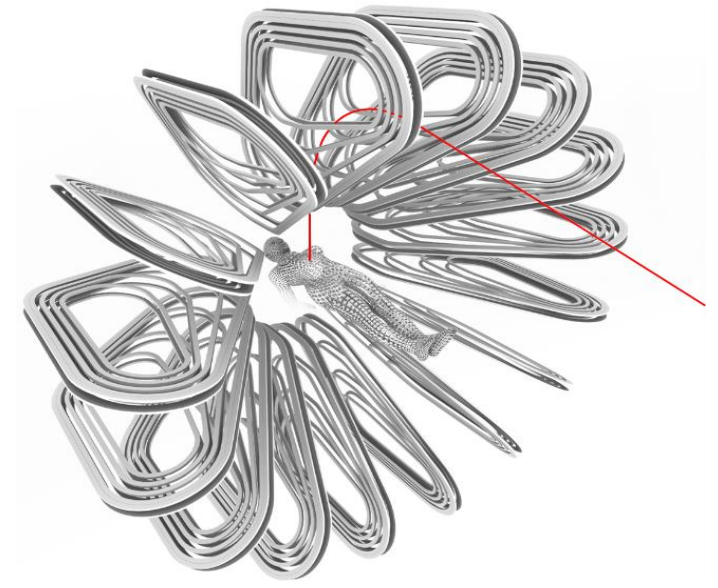

Fig. 1. Artistic representation of the 16 coils GaToroid for protons, with the patient inside the bore for size comparison (courtesy of Daniel Dominguez, CERN Design and Visual Identity Service) 
In [6] the ideal coil shape was derived, based on the simplification of a hard edge magnet, with uniform and constant field within the coils. In practice, the magnetic field in a conventional toroid is inversely proportional to the radius. Thus, to create a suitable magnetic field, able to bend the particle trajectories toward the isocenter in the whole range of treatment energies, it is necessary to modify the current distribution inside the coils, i.e. coil grading. Furthermore, the effect of fringe fields on the beam is not negligible and the hard edge approximation is not sufficient.

To find an appropriate solution, an iterative optimization, based on the minimization of the gap between the particle orbit positions at the isocenter at different energies, was used. The particle orbits identify the ideal trajectories around which the classical beam optics formalisms are defined [7]. A detailed beam dynamics analysis is beyond the scope of this manuscript and it described in [8]. The whole range of treatment energies for proton, i.e. $70 \mathrm{MeV}$ to $250 \mathrm{MeV}$, was analyzed and the convergence criterion was set to $1 \mathrm{~mm}$ at the isocenter. To maintain the orbit positioning at isocenter within $1 \mathrm{~mm}$, the vector magnet need a precision of about $5 \mathrm{mrad}$, while misalignment errors in the coils, and respective grades, must be limited below the $\pm 0.5 \mathrm{~mm}$ in the three axes.

The optimization was performed on the spacing between grades and on the torus parameters, such as the ideal field magnitude, internal radius and the position of the vector magnet. The analytical coil profile has a limb with negative curvature [6]. To simplify the winding procedure, the profile has been straightened, with little effect on the beam bending properties. The size of the coil is considered a crucial parameter, not only for the total footprint and weight of the gantry, but also for the amount of conductor and the stored energy, i.e. the cost of the machine [9]. For this reason, the geometry of the return current limb (located in the positive part of the z-axis in Fig.2) has been tilted to reduce the coil area and the length of conductor. Finally, to decrease the peak magnetic field on the conductors down to approximately $8 \mathrm{~T}$, gaps of the order of $2 \mathrm{~cm}$ have been introduced between grades in the outward leg. The coil geometry obtained by the optimization for a 16-coils GaToroid configuration, including current leads and grade jumps, is presented in Fig. 2. Each coil is wound as a double pancake and each layer is composed of 5 grades.

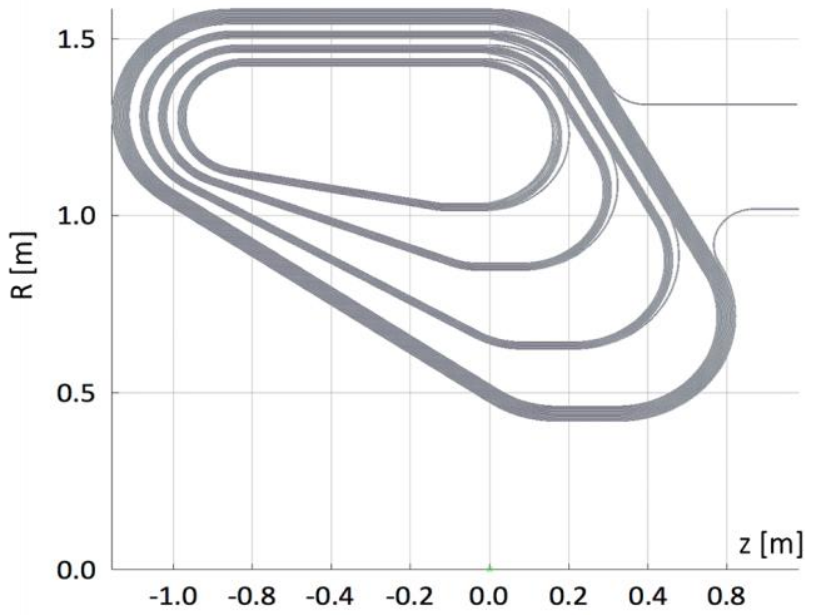

Fig. 2. Optimized coil geometry for proton GaToroid, including current leads and grade jumps
The core of electromagnetic simulations is based on Field2017 [10]. Dedicated software was developed for particle tracking and coil optimization. It is worth to underline that, because of the axis-symmetric configuration, the magnetic field on the patient, laying in the gantry bore, is below any value of concern for instrumentation or humans (order of $\mu \mathrm{T}$ ).

\section{CONDUCTORS AND QUENCH PROTECTION}

The main parameters of the optimized torus and coil are listed in Tab. I and Tab. II, respectively. The design of the machine was optimized limiting the peak magnetic field on the conductors in the order of $8 \mathrm{~T}$, to accommodate the use of both Low (LTS) and High (HTS) Temperature Superconductors.

TABLE I

PROTON GATOROID - TORUS MAIN PARAMETER

\begin{tabular}{lll}
\hline \hline Parameter & Units & Value \\
\hline Number of Coils & & 16 \\
Peak Magnetic Field & {$[\mathrm{T}]$} & 8.2 \\
Bore Diameter & {$[\mathrm{m}]$} & 0.8 \\
External Diameter & {$[\mathrm{m}]$} & 3.3 \\
Torus Length & {$[\mathrm{m}]$} & 1.8 \\
Vector Magnet Position & {$[\mathrm{m}]$} & 3.5 \\
Mass (coil + structure) & {$[$ tons $]$} & 12 \\
Stored Energy & {$[\mathrm{MJ}]$} & 34 \\
\hline \hline
\end{tabular}

TABLE II

PRoton GATOROID - COIL MAIN PARAMETER

\begin{tabular}{lll}
\hline \hline Parameter & Units & Value \\
\hline Number of Grades/Pancake & & 5 \\
Number of Pancakes & & 2 \\
Length & {$[\mathrm{m}]$} & 1.8 \\
Height & {$[\mathrm{m}]$} & 1.2 \\
Ampere-Turn & {$[\mathrm{MA}-\mathrm{turn}]$} & 1.4 \\
Operating Current & {$[\mathrm{kA}]$} & 1.8 \\
Cable Length & {$[\mathrm{km}]$} & 2.5 \\
Inductance (in the torus) & {$[\mathrm{H}]$} & 1.3 \\
\hline \hline
\end{tabular}

Given the low price, limited magnetic field ( $\sim \mathrm{T})$, simplicity of winding (no heat treatment) and high experience level gained in the last decades [11], Nb-Ti was chosen as LTS. Regarding HTS, between BSCCO and ReBCO the latter was selected. ReBCO coated conductors are widely used in the community, not only for toroidal fusion magnets [12][13], but also for new generation of high field magnets for particle physics [14] and medical applications, such as gantries [15] and NMR-MRI [16]. ReBCO is a costly material, produced with limited length and uniformity, and it is challenging to generate homogenous magnetic field due to the presence of screening currents. However, it does not require any heat treatment after the winding and, provided the absence of hard-way bending and small radii of curvature, can be easily wound. Finally, at the moment, coated conductors have larger margin of technological improvement [17] and represent a stimulating research challenge.

We have defined the cable parameters based on margin and hot-spot considerations, using an adiabatic approximation and lumped circuit parameters for the quench protection systems (i.e. no propagation considered). The operating current was chosen at a rather modest value, $1.8 \mathrm{kA}$, to reduce heat loads from the current leads. First, a solution with an external dump 
was considered, assuming two powering circuits of eight coils in series, 2 seconds for quench detection, a voltage limit at the coil terminals of $\pm 1 \mathrm{kV}$ and the cable parameters listed in Tab. III. During the current discharge, the hotspot temperature rise was evaluated both for LTS and HTS cables, and the results are shown in Fig. 3. The maximum temperature in the adiabatic condition is about $160 \mathrm{~K}$ and, therefore, in the presented approximation, the cable topologies are suitable for the magnet protection with an external dump.

TABLE III

PROTON GATOROID - LTS AND HTS CABLE

\begin{tabular}{llll}
\hline \hline Parameter & Unit & LTS & HTS \\
\hline Superconductor & & Nb-Ti & ReBCO \\
Cable & & Rutherford & Non-Twisted Stack \\
N strand/tape & 36 & 3 \\
Cable width & {$[\mathrm{mm}]$} & 12.2 & 12.2 \\
Cable thickness & {$[\mathrm{mm}]$} & 1.4 & 1.7 \\
Cu: Non-Cu & & 3 & 7 \\
Stabilizer & & Cu Profile & Co-Wound Cu \\
Impregnation & & Epoxy & Epoxy \\
Operating Current $-I_{o p}$ & {$[\mathrm{kA}]$} & 1.8 & 1.8 \\
Operating Temperature & {$[\mathrm{K}]$} & 4.2 & 20 \\
Peak magnetic Field & {$[\mathrm{T}]$} & 8.2 & 8.2 \\
Eng. Current Density & {$\left[\mathrm{A} / \mathrm{mm}^{2}\right]$} & 105 & 90 \\
\hline \hline
\end{tabular}

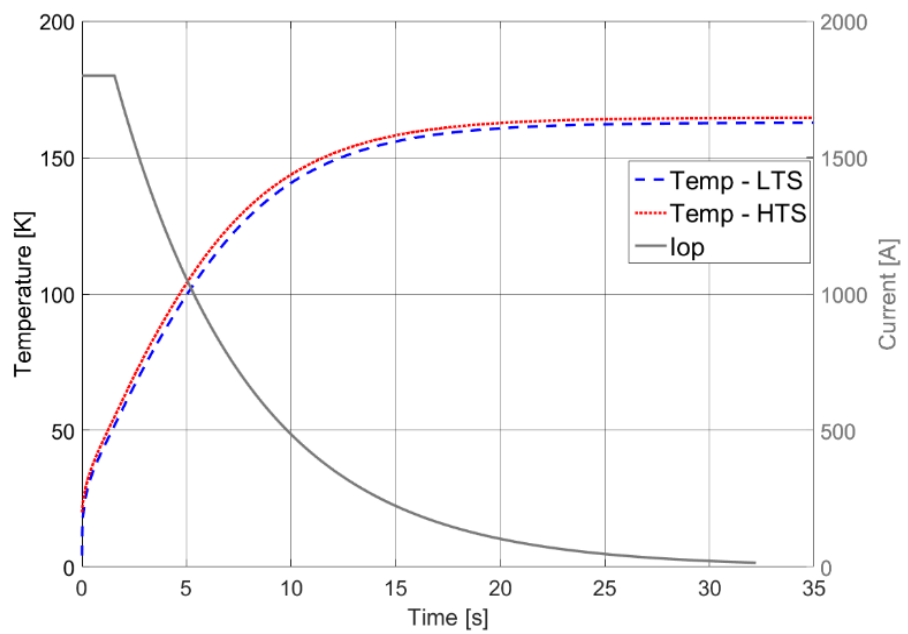

Fig. 3. Temperature of LTS (red dotted) and HTS (blue dashed) cable as a function of time, in case of external dump during the quench. Iop (gray solid) is the operating current in the magnet.

A second protection option was evaluated, considering the coils powered in series, by-passed by diodes, and quench heaters on the magnets [18]. Assuming 500 milliseconds of quench detection time, 190 Joules of energy introduced by the heaters on the whole coil and the parameters of Tab III, the hotspot temperature on the LTS cable is about $100 \mathrm{~K}$ and the magnet can be considered safely protected. However, since the energy margin of HTS in the operating conditions listed in Tab III is much larger, and the quench propagation much slower, the energy required at the heaters would exceed one hundred $\mathrm{kJ}$ per coil (about $15 \mathrm{~kJ}$ per grade). Therefore, for this magnet configuration we exclude an internal protection system for HTS. Nevertheless, given the steady-state configuration of GaToroid, HTS self-protected magnets, consisting of non or partially insulated windings [19][20], represent a further quench protection option to be evaluated in the future.

\section{Mechanical Design}

Given the magnetic field on the conductor surface, $B$, and the current density $J$, it is possible to calculate the force density distribution and integrate it on the conductor volume to obtain the total electromagnetic force generated by each coil $F_{c}$ :

$$
F_{c}=\iiint J \times B d V
$$

As expected from a toroidal magnet, $F_{c}$ is a centering force, acting radially on each coil toward the center of the torus [21].The forces are symmetric and uniformly distributed along the azimuthal direction $\varphi$. A first estimation of the supporting cylindrical structure was done, considering the hoop stress $\sigma_{\theta}$ created by a uniform pressure on a thin-walled cylindrical surface:

$$
\sigma_{\theta}=\frac{N_{\text {coils }} F_{c}}{2 \pi l t}
$$

where $N_{\text {coils }}$ is the number of coils, and $l$ and $t$ are respectively the length and the thickness of the bucking cylinder. In Fig. 4, the centering force $F_{c}$ and bucking cylinder structure are represented. The figure shows one-fourth of the symmetric toroidal structure, and it is worth noting the cavities on the cylinder, in between the coils, to accommodate the beam passage.

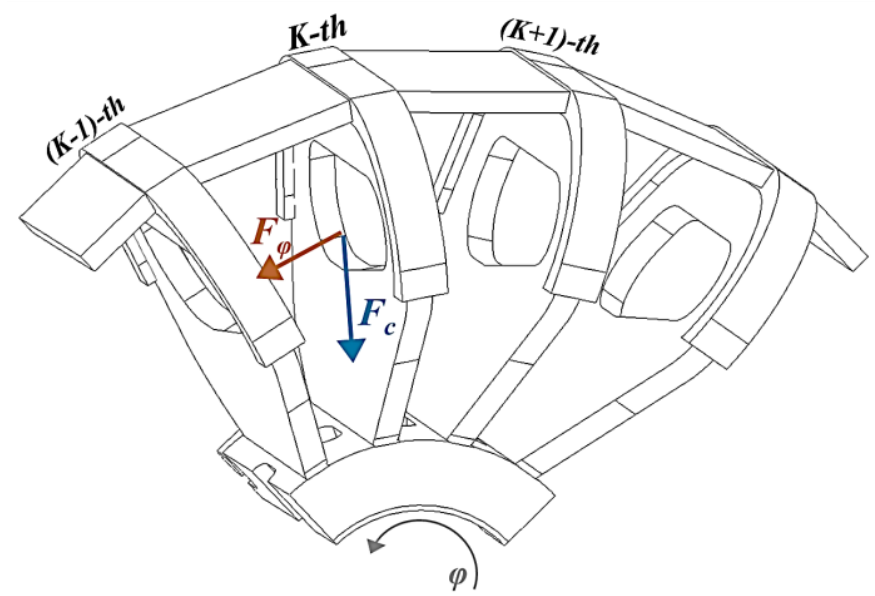

Fig. 4. Schematic representation of the prelaminar GaToroid mechanical structure, together with the force acting on the $k$-th coil. Centering force $F_{c}$ (blue arrow) in nominal operating conditions and overturning force $F_{\varphi}$ (red arrows), in case of a single coil quench $((k+1)$-th coil).

Similar calculations can be done in case of a coil quench. No transient phenomena were taken into account and the current was set constant and equal to zero on the quenched coil. Considering the coils plotted in Fig. 4, we can analyze the force acting on the $k$-th coil during the $(k+1)$-th quench. This overturning force $F_{\varphi}$ pushes the $k$-th coil toward the $(k-1)$-th in the azimuthal direction $\varphi$, i.e. out of the coil plane. $k$-th coil is experiencing the maximum overturning force during the quench of $(k+1)$-th one [22]. The structure foreseen to support the coils in case of quench is based on flat plates connecting the coils along the azimuthal direction $\varphi$. For a given length and thickness, the stress on this simplified geometrical structure can 
be evaluated. The force $F_{\varphi}$ generated in the case of quench and the inter-coil concept are schematically represented in Fig. 4.

The dimensions of the mechanical structures and the stresses are listed in Tab. IV, both for the bucking cylinder and the inter-coils structure. Finally, this preliminary mechanical design allows estimating the mass of the system, comprising the coils, casings, bucking cylinder and inter-coil structures. Assuming the use of stainless steel, the total weight of the assembly results in about 12 tons, i.e. one order of magnitude lighter than the nowadays available commercial gantries for proton therapy.

TABLE IV

PROTON GATOROID - FORCES AND STRUCTURE

\begin{tabular}{lll}
\hline \hline Parameter & Unit & Value \\
\hline Centering Force $\left|F_{c}\right|$ & {$[\mathrm{MN}]$} & 1.43 \\
Fault Force $\left|F_{\varphi}\right|$ & {$[\mathrm{MN}]$} & 1.92 \\
Cylinder thickness & {$[\mathrm{m}]$} & 0.06 \\
Cylinder length & {$[\mathrm{m}]$} & 0.5 \\
Inter-coil thickness & {$[\mathrm{m}]$} & 0.06 \\
Inter-coil length & {$[\mathrm{m}]$} & 0.5 \\
Cylinder hoop stress $\sigma_{\theta}$ & {$[\mathrm{MPa}]$} & 121 \\
Inter-coil stress $\sigma_{\varphi}$ & {$[\mathrm{MPa}]$} & 63 \\
\hline \hline
\end{tabular}

\section{HTS DEMONSTRATOR}

A single coil scaled-down demonstrator was designed to evaluate the practical feasibility of the coil and identify the main issues. The demonstrator is reduced in size by a factor 3 with respect to the GaToroid coil described so far. At nominal current, a single prototype coil will produce about $5 \mathrm{~T}$ (vs. $8 \mathrm{~T}$ in the full-size toroid) with force and stress distribution not representative of the final configuration. For this reason, we plan to test at over-current, to simulate internal forces, and we are evaluating the possibility of using a second identical coil with a magnetic mirror to reproduce centering and out-of-plane forces.

The demonstrator will be wound in HTS, to evaluate the cable configuration and quench protection system described above. The asymmetric geometry of the coils, its grades, layers, and respective connections, are challenges that can be tackled during the prototype winding. Finally, the demonstrator will serve as a benchmark of the electromagnetic design, through field mapping, and of the circuital model of the coil. Though not yet finalized, this will be particularly relevant in case a noninsulated or partially insulated winding is pursued.

TABLE V

HTS DEMONSTRATOR PARAMETERS

\begin{tabular}{lll}
\hline \hline Parameter & Units & Value \\
\hline Number of Grades & & 5 \\
Number of Layers & & 2 \\
Dimension Scale & & $1: 3$ \\
Cable & {$[\mathrm{m}]$} & HTS - ReBCO \\
Cable Length & & 290 \\
Impregnation & {$[\mathrm{kA}-$ turn $]$} & Epoxy \\
Ampere-Turn & {$[\mathrm{A}]$} & 470 \\
Operating Current & {$[\mathrm{K}]$} & 20 \\
Operating Temperature & {$[\mathrm{T}]$} & 5.1 \\
Peak Field &
\end{tabular}

The circuital model in this case is similar to the one proposed in [22], composed by 5 blocks of inductances and resistances per layer. The design choices for the demonstrator, described above, are summarized in Tab V. Work towards the demonstrator construction has started. A first dummy coil wound with stainless-steel tapes on glass-filled nylon spacers has been assembled, in order to verify the winding procedure and identify possible inaccuracies and faults in the design. Fig. 5 shows the first layer of the dummy GaToroid demonstrator, together with the ad hoc developed tooling and winding supports.

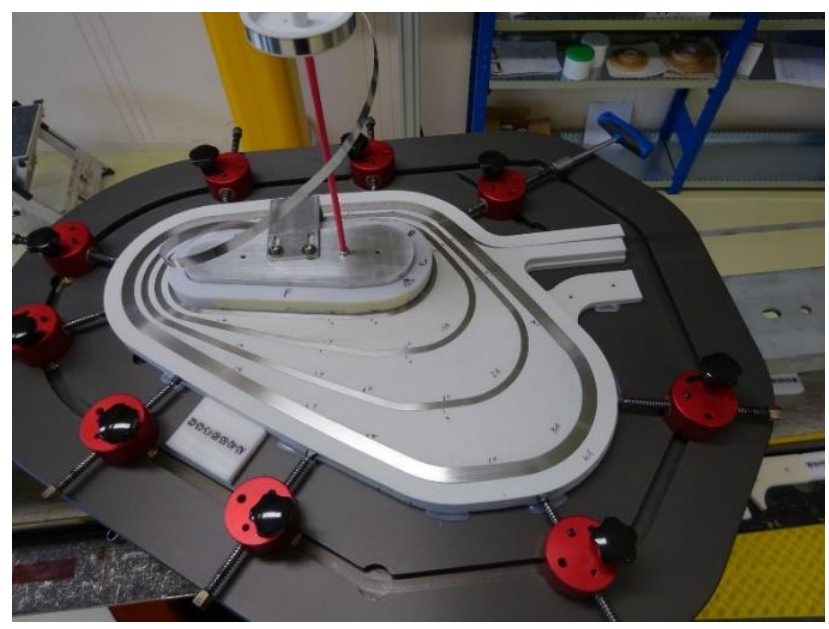

Fig. 5. First layer of GaToroid dummy prototype, wound in stainless steel tape on glass-filled nylon spacers (in white).

\section{CONCLUSION}

GaToroid is a novel concept of toroidal steady-state gantry for hadron therapy. This work presents the first magnetic design of the 16 coils GaToroid for protons. The coils were designed and optimized through a complete integration of magnetic field map calculation and bi-dimensional particle tracking. On the resulting torus, preliminary magnet design and studies on mechanical structures and quench protection were performed to evaluate the feasibility of the machine. A scaleddown HTS demonstrator of a single coil was designed and the first prototype in stainless steel tapes on glass-filled nylon spacers was wound. Further investigations are necessary to evaluate the gantry feasibility, including detailed analyses of integrated vacuum and cryogenic systems. They may be composed of $N_{\text {coils }}$ flat vacuum pipes originating from the vector magnet and entering the cryostat that encloses the whole torus except for the central bore. Although the engineering design of GaToroid superconducting torus is just at the beginning, the concept seems very promising and could result in a significant reduction of gantries size and weight, facilitating societal penetration of hadron therapy.

\section{ACKNOWLEDGMENT}

Jacky Mazet, Sebastien Clement, Frederic Garnier, Jeremie Massard and Juan Carlos Perez from CERN TE-MSC-MDT section are gratefully acknowledged for the past, present and future prototyping work. 


\section{REFERENCES}

[1] A. Degiovanni and U. Amaldi, "History of hadron therapy accelerators," Physica Medica. 2015.

[2] S. Yan, H.-M. Lu, J. Flanz, J. Adams, A. Trofimov, and T. Bortfeld, "Reassessment of the Necessity of the Proton Gantry: Analysis of Beam Orientations From 4332 Treatments at the Massachusetts General Hospital Proton Center Over the Past 10 Years," Int. J. Radiat. Oncol., vol. 95, no. 1, pp. 224-233, May 2016.

[3] T. Haberer, J. Debus, H. Eickhoff, O. Jäkel, D. Schulz-Ertner, and U. Weber, "The heidelberg ion therapy center," Radiother. Oncol., vol. 73, pp. S186-S190, Dec. 2004.

[4] S. Mori et al., "Design of a superconducting rotating gantry for heavy-ion therapy," Phys. Rev. Spec. Top. Accel. Beams, vol. 15, 2012.

[5] L. Bottura, "A Gantry and apparatus for focussing beams of charged particles," Patent WO2019/224215, 2019, https://worldwide.espacenet.com/patent/search/family/062235811/publica tion/WO2019224215A1?q=WO2019\%2F224215\&called_by=epo.org

[6] L. Bottura and E. Felcini, "Novel Concept of a Gantry for Hadron Therapy Based on Steady Toroidal Magnets," CERN Internal Report, unpublished, https://edms.cern.ch/document/1864555/2. To be submitted to Nucl. Instrum. Methods.

[7] J. Lawson, The Physics of Particle Accelerators, vol. 34, no. 1. Oxford Univeristy Press Inc., 1984.

[8] E. Felcini, L. Bottura, J. Van Nugteren, A. Gerbershagen and B. Dutoit, "Particle Tracking and Beam Optics Analysis on Toroidal Gantry for Hadron Therapy," CERN Internal Report, unpublished, https://edms.cern.ch/document/2116152/0. To be submitted to Phys. Rev. Accel. Beams

[9] M. A. Green and B. P. Strauss, "The cost of superconducting magnets as a function of stored energy and design magnetic induction times the field volume," IEEE Trans. Appl. Supercond., vol. 18, no. 2, pp. 248-250, 2008.

[10] J. van Nugteren, "Software Development for the Science and Design behind Superconducting Magnet Systems," Cern Internship Report

[11] L. Rossi, "Superconducting cable and magnets for the large hadron collider," LHC Proj. Rep. 694, no. March 2004, pp. 14-18, 2003.

[12] A. Sykes, "Compact fusion energy based on the spherical tokamak," Nucl. Fusion, vol. 58, no. 1, 2018.

[13] D. G. Whyte, J. Minervini, B. LaBombard, E. Marmar, L. Bromberg, and M. Greenwald, "Smaller \& Sooner: Exploiting High Magnetic Fields from New Superconductors for a More Attractive Fusion Energy Development Path," J. Fusion Energy, vol. 35, no. 1, pp. 41-53, 2016.

[14] G. A. Kirby et al., "First Cold Powering Test of REBCO Roebel Wound Coil for the EuCARD2 Future Magnet Development Project," IEEE Trans. Appl. Supercond., vol. 27, no. 4, pp. 1-7, 2017. Art. No. 4003307

[15] S. Takayama et al., "Design of conduction-cooled HTS coils for a rotating gantry," Phys. Procedia, vol. 67, pp. 879-884, 2015.

[16] J. Bascunan, Seungyong Hahn, Youngjae Kim, Jungbin Song, and Y. Iwasa, "90-mm/18.8-T All-HTS Insert Magnet for $1.3 \mathrm{GHz}$ LTS/HTS NMR Application: Magnet Design and Double-Pancake Coil Fabrication," IEEE Trans. Appl. Supercond., vol. 24, no. 3, pp. 1-4, 2013. Art. No. 4300904

[17] D. Uglietti, "A review of commercial high temperature superconducting materials for large magnets: From wires and tapes to cables and conductors," Supercond. Sci. Technol., vol. 32, no. 5, 2019.

[18] A. S. and F. S. F. Rodriguez-Mateos, P. Pugnat, S. Sanfilippo, R. Schmidt, "Quench Heater Experiments on the Lhc Main Superconducting Magnets," LHC Proj. Rep. 418, no. September 2000.

[19] S. Hahn, D. K. Park, J. Bascuñán, and Y. Iwasa, "HTS pancake coils without turn-to-turn insulation," IEEE Trans. Appl. Supercond., vol. 21, no. 3 PART 2, pp. 1592-1595, 2011.

[20] Y. I. S. Hahn, K. Kim, K. Kim, H. Lee, "Current Status of and Challenges for No-Insulation HTS Winding TechniqueCurrent Status of and Challenges for No-Insulation HTS Winding Technique," Teion Kogaku (Journal Cryog. Supercond. Soc. Japan), vol. 53, no. 1, pp. 2-9, 2018.

[21] R. J. Thome and J. M. Tarrh, MHD and fusion magnets. John Wiley \& Sons Inc, 1982.

[22] Y. Oga, S. Noguchi, and H. Igarashi, "Overturning force simulation on chain of quenches of toroidal HTS-SMES," IEEE Trans. Appl. Supercond., vol. 22, no. 3, p. 4701904, 2012. Art. No. 4701904

[23] K. R. Bhattarai, K. Kim, S. Kim, S. Lee, and S. Hahn, "Quench analysis of a multiwidth no-insulation 7-T 78-mm REBCO magnet," IEEE Trans. Appl. Supercond., vol. 27, no. 4, pp. 1-5, 2017. Art. No. 4603505 